\title{
Enabling Implicit Human Computer Interaction A Wearable RFID-Tag Reader
}

\author{
Albrecht Schmidt and Hans-W. Gellersen \\ $\mathrm{TecO}$, University of Karlsruhe \\ 76131 Karlsruhe, Germany \\ Phone +49 721 6902-29 \\ \{albrecht | hwg\}@teco.edu
}

\begin{abstract}
Implicit human computer interaction is based on the concept of using user activity in the real world as input to computers. Implicit HCI can help to reduce the problem of user input to wearable computers. In this paper we report on wearable RFID technology that facilitates applications that are triggered by handling tagged physical objects. We also report on a case study in which the technology was integrated with an enterprise resource planning system to optimize work processes that involve physical objects.
\end{abstract}

\section{Introduction}

In traditional computing hard- and software is mainly designed to support users in office environments (e.g. desktop computers with keyboard and mouse). Human computer interaction (HCI) is based on an explicit metaphor - the users explicitly request actions that should be performed by the computer.

In wearable computing it is much more difficult to provide input to the system. Most available input devices for explicit interaction either carry a high cognitive load (e.g. Twiddler), are hardly usable when the user is moving (e.g. pen input, arm mounted keyboards, pointing devices), or have significant performance problems in real-world deployment (e.g. speech recognition).

In the case studies described in this paper we explore implicit HCI using RFIDs and a wearable tag reader. The term implicit $H C I$ describes user actions that are not primarily aimed to interact with a computer, but which are recognized by the computer and used as input, see [2] for an in-depth discussion.

In many work situations physical goods are at the center of the tasks that workers perform. We made the following assumption to enable implicit HCI: knowledge of which physical objects are handled and of the tasks that determine a work situation enables the system to reason on the intention of the user.

\section{RFID Tags and Readers}

RFID systems consist of tags and readers. These technologies have been widely applied over recent years for identification and tracking applications.

\author{
Christian Merz \\ SAP AG, Corporate Research, CEC Karlsruhe, \\ 76131 Karlsruhe, Germany \\ Phone +49 $7216902-73$ \\ christian.merz@sap.com
}

Passive RFID tags are small electronic components with an integrated circuit and a small antenna usually sealed in one small package, see figure $1 \mathrm{a}$ ). The tags do not need a battery; they are energized during access by the reader via electromagnetic induction.

The reader is an electronic component that supplies energy to the tags for a short time and then communicates with the tag.

There are many different types of RFID systems. They can be discriminated by:

- The operating range (distance between the coil of the reader and the tag)

- Access Mode (read only, read/write)

- Protocol (anti-collision to read tags simultaneously)

- Frequency used

RFID tags are considered in many areas (e.g. logistics, sales) as a replacement of barcode in the future. Currently they are still more expensive than barcode and must be attached instead of printed, but they overcome many of the limitations of barcode.

In [3] it is discussed how RFIDs can be used to bridge physical and virtual worlds.

\section{A Wearable Tag Reader}

For our case studies we build a wearable tag reader as shown in Figure $1 \mathrm{c}$ ). We also made different types of coils that are integrated into clothing or worn on the body, see Figure $1 \mathrm{~b}$ ).

For our system we detached the coil from an EasyKey ${ }^{\mathrm{TM}}$ transponder reader module and added a power regulation and a TTL to RS232 converter to the module. The module we used is read-only, works on $125 \mathrm{kHz}$, and offers a maximal reading range of 3 inch. The reader is integrated into a 2.5 by 4 inch housing with a belt clip. The module is switched into continuous read mode - over the time period a tag is detected near the coil, its ID is repeatedly propagated over the serial line. There is also a connector for the coil housing.

We build and used different coils with the reader, which have been connected with a cable to the tag-reader. The coil used in the case study described below is made of a flexible wire sewn into a work glove. The usage scenario determines the type of coil and its placement in clothing. The wearable computer is connected via serial line to the reader module. 


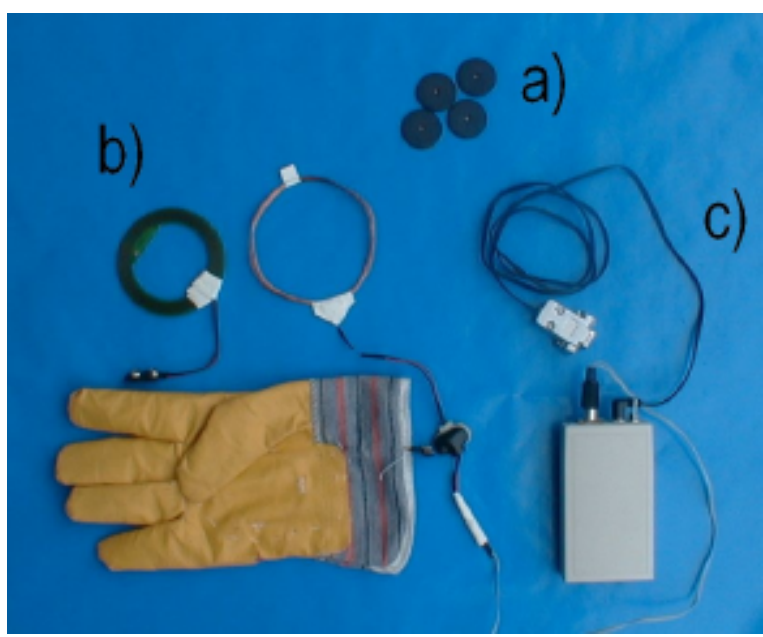

Figure 1: a) RFIDs, b) Different Coils, c) The Wearable Tag Reader.

\section{Software - Giving Meaning to Tags}

Each tag has a unique id consisting of 11 hex characters. The reader sends this string continuously over the serial line to the wearable computer while a tag is nearby. To build systems that are flexible and can easily make use of the information we decided to implement software that maps RFIDs to URLs on the WWW.

The software has three parts: a module that listens on the serial port, a web browser component, and a mapping table. When a ID appears on the serial line this ID is mapped to an URL, then the web browser is called with this URL. The software is implemented using Visual Basic and run on Windows based systems.

To illustrate how the system can be used consider the following application. On a web server is a CGI-script that increases a counter every time it is accessed (similar to well known counters on web pages). The mapping table is initialized so that for any RFID this URL is called. When now handling tagged physical objects the RFID is read automatically then the associated URL is called and the counter is increased.

\section{Case Studies}

We have used this system to explore implicit human computer interaction based on RFID tags. The first case describes an implementation of real world bookmarks. The second shows how complex business processes can be simplified using the suggested technology.

\subsection{Real World Bookmarks}

Physical objects have often a specific meaning to the user. When their identity is associated with a URL, objects can serve as real-world bookmarks. We have explore a range of examples that employ object/URL mappings:
- Objects to trigger applications: pick up pen $\rightarrow$ open editor by calling a URL with an empty document

- Objects as bookmark to information: wooden spoon $\rightarrow$ suggesting a recipe

- Personal object to access individualized information: wallet $\rightarrow$ show user's stock portfolio

In [1] similar approaches have been suggested using barcode; in [3] real world bookmarks based on electronics tags are discussed.

\subsection{Integration with mySAP.com}

The tag reading system is integrated with mySAP.com enterprise resource planning components like R/3 using the middleware product SAP Business Connector. By mapping the tag IDs to specific URLs the integration of business functionality accessible by remote enabled function calls (BAPI's) and IDocs (Intermediate Documents) is implemented. The output format is either XML or HTML that can be displayed by the browser.

The wide range of typical applications includes data reading/recording in inventory management, warehouse management, production planning, logistics execution or quality management. As an example an employee equipped with a wearable RFID reader can check incoming deliveries against the purchase order stored in SAP R/3 by reading the tag that acts as a packing list. If the check has been successful the goods receipt could be automatically posted. In another scenario a quality inspector records inspection results in SAP R/3 by sorting out bad parts.

\section{Conclusion}

In the paper we argued in favor of implicit HCI to address the problem of user input to wearable computers. We described a hardware and software implementation of a wearable tag reading system enabling implicit HCI. In this system RFID tags attached to physical objects are associated with URLs. This mechanism makes it easy to build applications using standard web technology or to integrate the RFID system with existing systems, such as SAP/R3.

\section{References}

[1] P. Ljungstrand, L.E. Holmquist. WebStickers: Using Physical Objects as WWW Bookmarks. Extended Abstracts of the CHI 99, Pittsburg, USA 1999. pp 332-333.

[2] A. Schmidt. Implicit Human Computer Interaction Through Context. Personal Technologies, Vol 4(2), June 2000.

[3] R. Want, K.P. Fishkin, A. Gujar, B.L. Harrison. Bridging Physical and Virtual Worlds with Electronic Tags. Proc. of the CHI 99, Pittsburg, USA 1999. pp 370-377. 